\title{
Early combination therapy with hydroxychloroquine and azithromycin reduces mortality in 10,429 COVID-19 outpatients
}

Matthieu MILLION ${ }^{1, *}$, Jean-Christophe LAGIER ${ }^{1}$, Hervé TISSOT-DUPONT ${ }^{1}$, Isabelle RAVAUX ${ }^{1}$, Catherine DHIVER ${ }^{1}$, Christelle TOMEI ${ }^{1}$, Nadim CASSIR $^{1}$, Léa DELORME ${ }^{2}$, Sébastien CORTAREDONA ${ }^{2}$, Sophie AMRANE ${ }^{2}$, Camille AUBRY², Karim BENDAMARDJI ${ }^{1}$, Cyril BERENGER ${ }^{2}$, Barbara DOUDIER ${ }^{1}$, Sophie EDOUARD ${ }^{1}$, Marie HOCQUART $^{2}$, Morgane MAILHE $^{1}$, Coralie PORCHETO ${ }^{1}$, Piseth SENG ${ }^{1}$, Catherine TRIQUET ${ }^{1}$, Stéphanie GENTILE ${ }^{3}$, Elisabeth JOUVE ${ }^{3}$,

Audrey GIRAUD-GATINEAU ${ }^{2}$, Herve CHAUDET ${ }^{2}$, Laurence CAMOIN-JAU ${ }^{4}$, Philippe COLSON ${ }^{1}$, Philippe GAUTRET ${ }^{2}$,

Pierre-Edouard FOURNIER ${ }^{2}$, Baptiste MAILLE ${ }^{5}$, Jean-Claude DEHARO ${ }^{5}$, Paul HABERT $^{6}$, Jean-Yves GAUBERT ${ }^{6}$,

Alexis ]ACQUIER ${ }^{6}$, Stéphane HONORE ${ }^{7}$, Katell GUILLON-LORVELLEC ${ }^{1}$, Yolande OBADIA ${ }^{2}$,

Philippe PAROLA ${ }^{2}$, Philippe BROUQUI ${ }^{1}$, Didier RAOULT ${ }^{1}$

${ }^{1}$ IHU-Méditerranée Infection, Aix Marseille Univ, Assistance Publique Hôpitaux de Marseille (AP-HM), Institut de recherche pour le développement (IRD) Unité Microbes Evolution Phylogénie et Infections (MEPHI),13005 Marseille, France

${ }^{2}$ IHU-Méditerranée Infection, Aix Marseille Univ, Assistance Publique Hôpitaux de Marseille (AP-HM), Institut de recherche pour le développement (IRD), Unité Vecteurs - Infections Tropicales et Méditerranéennes (VITROME), Service de santé des armées (SSA), 13005 Marseille, France

${ }^{3}$ EA 3279: CEReSS - Health Service Research and Quality of Life Center, Service d'Evaluation Médicale, Aix Marseille Univ, Assistance Publique Hôpitaux de Marseille, 13005 Marseille, France

${ }^{4}$ Laboratoire D'Hématologie, Hôpital de La Timone, Assistance Publique Hôpitaux de Marseille, 13005 Marseille, France

${ }^{5}$ Service de Cardiologie, Centre Hospitalier Universitaire La Timone, Assistance Publique Hôpitaux de Marseille, Aix Marseille Univ, C2VN, 13005 Marseille, France

${ }^{6}$ Radiology Department, La Timone Hospital, Assistance Publique Des Hôpitaux de Marseille, Aix Marseille Univ, LIIE, CERIMED, 13005 Marseille, France ${ }^{7}$ Service de Pharmacie, Hôpital Timone, Laboratoire de Pharmacie Clinique, Aix Marseille Université AP-HM, 13005 Marseille, France

*Correspondence: matthieu.million@gmail.com (Matthieu MILLION)

DOI:10.31083/j.rcm2203116

This is an open access article under the CC BY 4.0 license (https://creativecommons.org/licenses/by/4.0/).

Submitted: 10 August 2021 Revised: 27 August 2021 Accepted: 30 August 2021 Published: 24 September 2021

We evaluated the age-specific mortality of unselected adult outpatients infected with SARS-CoV-2 treated early in a dedicated COVID19 day hospital and we assessed whether the use of hydroxychloroquine (HCQ) + azithromycin (AZ) was associated with improved survival in this cohort. A retrospective monocentric cohort study was conducted in the day hospital of our center from March to December 2020 in adults with PCR-proven infection who were treated as outpatients with a standardized protocol. The primary endpoint was 6-week mortality, and secondary endpoints were transfer to the intensive care unit and hospitalization rate. Among 10,429 patients (median age, 45 [IQR 32-57] years; 5597 [53.7\%] women), 16 died (0.15\%). The infection fatality rate was $0.06 \%$ among the $8315 \mathrm{pa}-$ tients treated with $\mathrm{HCQ}+\mathrm{AZ}$. No deaths occurred among the $8414 \mathrm{pa}-$ tients younger than 60 years. Older age and male sex were associated with a higher risk of death, ICU transfer, and hospitalization. Treatment with $\mathrm{HCQ}+\mathrm{AZ}(0.17$ [0.06-0.48]) was associated with a lower risk of death, independently of age, sex and epidemic period. Metaanalysis evidenced consistency with 4 previous outpatient studies $\left(32,124\right.$ patients-Odds ratio 0.31 [0.20-0.47], $\left.I^{2}=0 \%\right)$. Early ambulatory treatment of COVID-19 with $\mathrm{HCQ}+\mathrm{AZ}$ as a standard of care is associated with very low mortality, and HCQ+AZ improve COVID-19 survival compared to other regimens.

\section{Keywords}

SARS-CoV-2; COVID-19; Hydroxychloroquine; Azithromycin; Ambulatory; Outpatients; Treatment

\section{Introduction}

The SARS-CoV-2 pandemic infected 200 million people and killed 4.2 million people by August 4, 2021, corresponding to an overall infection fatality rate (IFR) of $2 \%$ [1]. Health agencies in Western countries have focused on contagion control measures (lockdown), late-stage hospitalized patients, intensive care units, and vaccination, but for reasons that are yet to be clarified, early treatment has not been emphasized [2-4]. In eastern countries such as China, India, Iran, and Saudi Arabia, where early treatment and prevention with repurposed antivirals, particularly hydroxychloroquine (HCQ), has been widely implemented [5-8], lower IFRs than Western countries, where early treatment with orally available molecules has been overlooked or even discouraged, have been reported [1]. In addition, countries using chloroquine or HCQ as a treatment from the start of the epidemic 
had a much slower dynamic in daily deaths [9]. The antiviral effect of chloroquine and its derivatives (HCQ) against SARSCoV-2 was identified as early as February 2020 through in vitro studies in early Chinese publications $[10,11]$ and a preliminary trial in our center [12]. The synergistic in vitro antiviral effect of the combination of HCQ with azithromycin (AZ) was further reported [13]. In addition, HCQ has several anti-inflammatory and antithrombotic properties [14], which is of particular interest in the context of COVID-19associated inflammation and coagulopathy.

Our previous observational study [15] reported a beneficial effect on thousands of cases, but in- and outpatients were not analyzed separately. The largest publicly available ambulatory studies included an Iranian study with 28,759 outpatients and a study in Saudi Arabia with 5541 outpatients, both evidencing a 4-fold reduced risk of death with HCQ $[5,6]$. The importance of earliness of treatment has also been recently emphasized by a Chinese study reporting that HCQ, when administered in the first 5 days after symptom onset, improves prognosis and reduces viral shedding [7]. The effect of early ambulatory treatment with HCQ combined with AZ on COVID-19 mortality has not been reported in a large series. Here, we evaluated the age-specific mortality of unselected adult outpatients infected with SARS-CoV-2 managed early in a dedicated COVID-19 day hospital offering standardized treatment based on HCQ+AZ. We also assessed whether the use of $\mathrm{HCQ}+\mathrm{AZ}$ was associated with improved IFR and lower rates of intensive care unit (ICU) admission and hospitalization in a conventional ward (HC) in this cohort. A meta-analysis of studies assessing early HCQ in COVID-19 outpatients was conducted to test consistency with available literature.

\section{Methods}

\subsection{Study design, setting and participants}

Detailed methods are provided in the Supplementary data (see Supplementary Methods). Briefly, this retrospective cohort study, reported according to the STROBE guidelines, was conducted in the day hospital of the Institut Hospitalo-Universitaire (IHU) Méditerranéee Infection (https://www.mediterranee-infection.com/), Assistance Publique-Hôpitaux de Marseille (AP-HM), southern France, with an inclusion period from 17 March to 31 December 2020, and follow-up until 11 February 2021.

All patients with a positive PCR test regardless of symptoms were proposed to come in our unit for treatment. We assessed the correlation between the Cycle threshold value (Ct) of quantitative real-time PCR (qPCR) and viral viability in culture for 3790 SARS-CoV-2 positive samples [16]. Using our culture protocol, 1941 were positive with an inverse correlation with the $\mathrm{Ct}$, while culture positivity rate was still $70 \%$ and $20 \%$ for Ct of 25 and 30, respectively. For Ct of 35 , only $3 \%$ of samples were positive in culture. In the present study, all included patients had a qPCR Ct $<35$.
Our center was the first to perform PCR screening since February, 2020 [17], whereas mass screening was generalized in France since summer, 2020. Thus, patients with a positive PCR or antigenic test performed outside our center including in private laboratories could come for treatment at IHU but were systematically confirmed by a rapid PCR test (time-toresult $<1 \mathrm{~h}$ ) in our center [18], before entering our unit. Our isolated unit with an uncontrolled negative pressure has been fitted out with an airlock, individual protective equipment, a forward path for patients with successive triage by measurement of vital parameters, a rapid point-of-care blood test for serum potassium and blood gas analysis (15 min), two ECG stations, consultation rooms as well as 8 beds to take care of patients in respiratory distress awaiting transfer to hospitalization unit or intensive care unit.

Age, sex, date of day hospital attendance, and treatment were collected for all patients. The presence of symptoms, the time between the onset of symptoms and day hospital attendance and the time between the positive PCR sample and day hospital attendance were collected only for a subgroup of patients who were managed in December 2020. Compared to previous retrospective cohort studies of our center [15], this study focused on outpatients with ambulatory treatment, namely, patients who presented with non-severe COVID-19 who returned home and were not immediately hospitalized in a conventional ward.

\subsection{COVID-19 management}

Detailed COVID-19 management and therapeutic protocol are provided in supplementary data. Briefly, patients were systematically administered HCQ at $200 \mathrm{mg}$ tid for 10 days, $\mathrm{AZ}$ at $500 \mathrm{mg}$ on day 1 and then $250 \mathrm{mg}$ for 4 days in the absence of contraindications. $\mathrm{HCQ}+\mathrm{AZ}$ was prescribed as offlabel medication. Anticoagulants were added after the death of a 60-year-old physician with no previous medical history who progressed despite $\mathrm{HCQ}+\mathrm{AZ}$ and died of severe coagulopathy, disseminated and pulmonary thrombosis, and on the basis of the literature $[19,20]$. Zinc was added to the treatment protocol following the publication of a clinical study reporting its beneficial effect in combination with HCQ [21], its beneficial effect in vitro, and the association between zinc deficiency and the lethality rate of COVID-19 [14]. Fig. 1 (Ref. $[22,23])$ shows the chronology of therapeutic changes.

\subsection{Outcomes}

The primary objective was to evaluate the age-specific 6week IFR of unselected adult outpatients infected with SARSCoV-2 who were managed early in a dedicated COVID19 day hospital offering standardized treatment based on HCQ+AZ. The secondary objective was to test whether the use of $\mathrm{HCQ}+\mathrm{AZ}$ was associated with improved IFR and lower ICU and HC rates in this cohort. The main considered confounding factors were age, sex, and epidemic period. The comprehensiveness of the HC cases, ICU transfers and deaths was optimized by using an automatic query of the informatic system of the APHM (Departement d'Information Médicale 


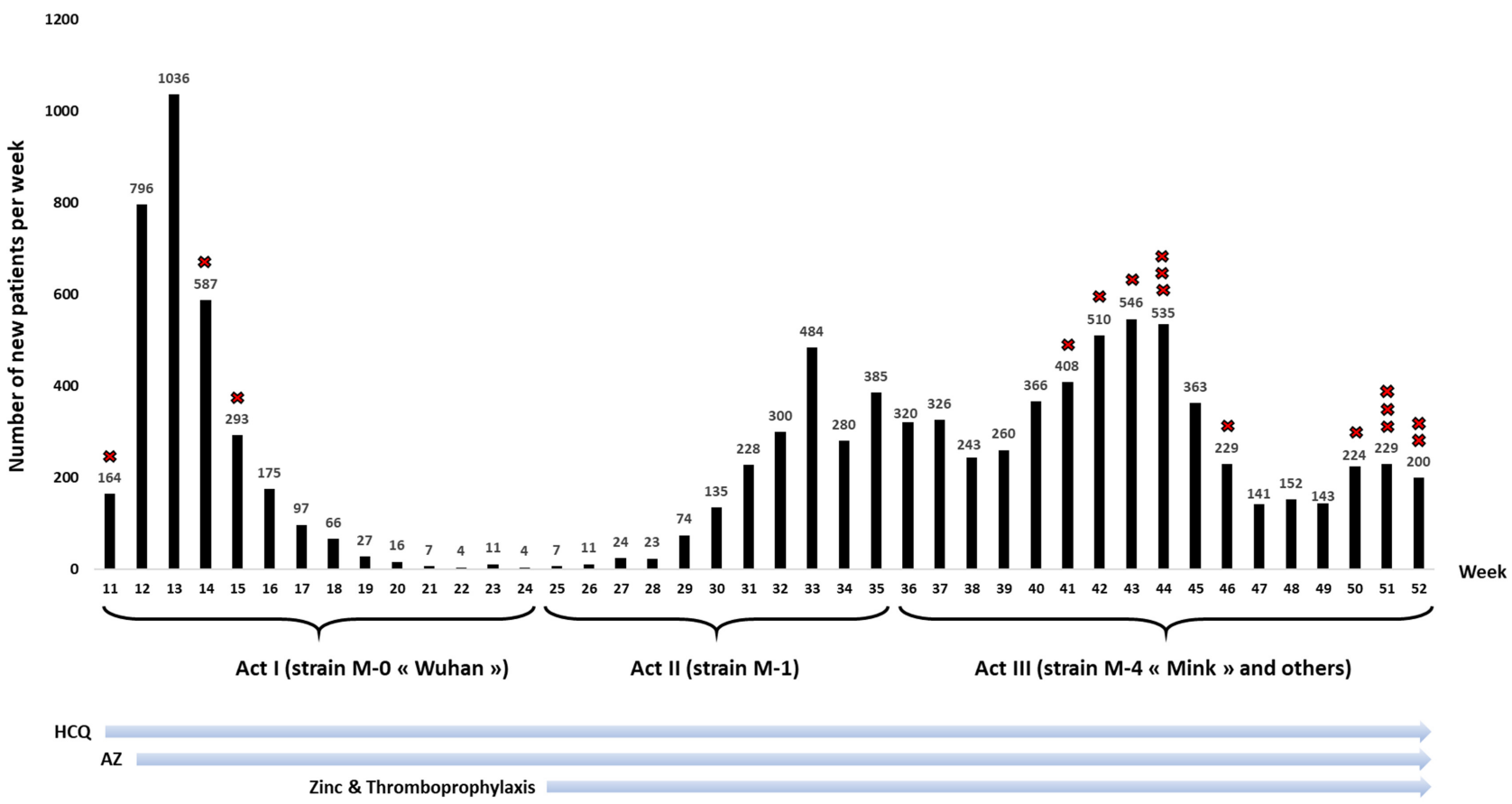

Fig. 1. Pandemic periods, changes in therapeutic protocol and number of ambulatory patients treated in our daycare hospital $(\mathrm{n}=10,429)$. HCQ, Hydroxychloroquine; AZ, Azithromycin. The three pandemic periods corresponded to different SARS-CoV-2 variants (Marseille-0 similar to the Wuhan strain for Act I, Marseille-1 for Act II, Marseille-4 and others for Act III-see [22, 23] and Supplementary data). Red crosses represent deaths.

(DIM)) and, for deaths only, the National Register of Deceased Persons (NRDP) accessed on March 2021, which included reported deaths for 2020 and January and February 2021 [24]. In agreement, the deaths were collected for all patients regardless of the place of death (in hospital or not) in France.

\subsection{Statistical analysis}

Associations between treatment $(\mathrm{HCQ}+\mathrm{AZ})$, age, sex and epidemic period, and clinical outcomes (deaths, ICU admissions, HC) were estimated using multivariable logistic regression with adjustments for age, sex and epidemic period. A two-sided $\alpha$ value of less than 0.05 was considered statistically significant. Analyses were carried out using SAS 9.4 statistical software (SAS Institute, Cary, NC, USA). Metaanalysis on outpatient treatment of COVID-19 with HCQ was performed using random effects modeling for odds ratios. Meta-analysis was performed using the $\mathrm{R}$ package meta.

\section{Results \\ 3.1 Participants}

In 2020, 11,725 COVID-19 patients were treated and followed in our day hospital. Among these, 504 were immediately hospitalized in the conventional ward and were excluded. Among 11,221 outpatients, 792 were excluded for the following reasons: 424 patients with unavailable information on treatment, 265 minor patients, 82 considered cured, and 72 without a positive PCR test (though one patient could have been excluded for more than one reason) (Fig. 2). None refused the use of their data. After exclusion of these patients, our ambulatory cohort included 10,429 outpatients.

The trend in the number of patients seen in the day hospital per week is shown in Fig. 1 and reflects 3 pandemic periods corresponding to different variants $[22,23]$. The median age was 45 [IQR 32-57] years, and 5597 [53.7\%] were women. Age and the sex ratio differed according to the epidemic period (Supplementary Table 1, Supplementary Fig. 1), with patients being older during the third period. The median delay from symptom onset to day hospital attendance was 4 days (interquartile range 2 to 6 days, information available for 1066 symptomatic patients seen in December 2020), and that from the screening positive test was 1 day (1-3 days, information available for 1119 patients). These delays were very similar among all age intervals (Supplementary Table 2). Among 1119 patients treated in December 2020, 53 (4.7\%) were asymptomatic at presentation.

Among the 10,429 included patients, 8315 received the combination therapy $\mathrm{HCQ}+\mathrm{AZ}$ (79.7\%), 1091 received AZ alone (10.5\%), 207 received HCQ alone (2.0\%-mainly the first week, Fig. 1), and 816 did not receive either HCQ or $\mathrm{AZ}$ (7.8\%). The reasons for not prescribing treatment are mentioned in Supplementary Table 3. No serious adverse events nor torsade de pointes was observed. Of these 10,429 patients, 21 had a second SARS-CoV-2 infection (0.2\%) with a median time to reinfection of 160 days (interquartile range 127 to 209 days). 
11,725 patients presenting at the IHU daycare hospital for COVID-19 in 2020

(March, 17 to December, 31)

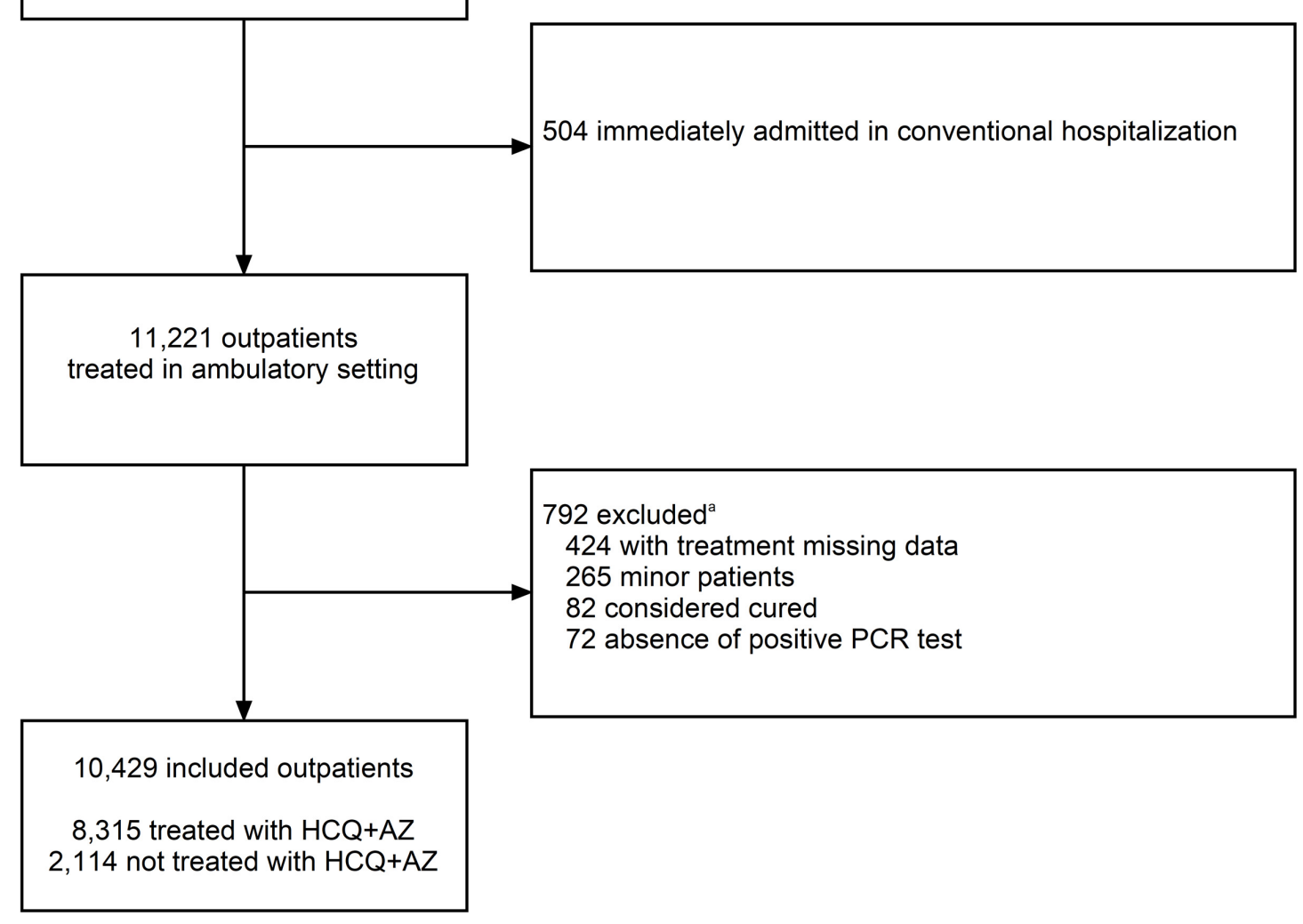

Fig. 2. Study flowchart. ${ }^{a}$ One patient may be excluded for more than one reason. IHU, Institut Hospitalo-Universitaire Méditerranée Infection; HCQ, hydroxychloroquine; AZ, azithromycin.

\subsection{Outcomes \\ 3.2.1 Deaths}

Among the 10,429 ambulatory patients, there were 16 deaths $(0.15 \%)$ (Table 1, Figs. 1,3. No patient under 60 years of age died $(0 / 8414(0 \%), 95 \%$ confidence interval $0.0 \%$ to 0.4\%) (Fig. 3). Therefore, the IFR among the 2015 patients aged 60 and over was $0.8 \%$. 11/16 deaths (70\%) were common to both data sources (DIM \& NRDP). Two were identified only with the DIM, and three were identified only with the NRDP. The median age of the decedents was 78 years (interquartile age 69-82 years), and 12/16 (75\%) were male. Thirteen $(81 \%)$ had a Charlson score $\geq 5$, corresponding to a risk of death within one year of more than $85 \%$, so that only three were expected not to die in the following year. Among 13 patients with a known cause of death, 12 presented with respiratory failure, 1 presented with anaphylactic and septic shock after dexamethasone, one presented with neurological failure, and 6 presented with severe coagulopathy. None of the deaths with a known cause were related to a side effect of hydroxychloroquine and/or azithromycin or a torsade de pointe.
There were 5 deaths among the 8315 patients who received HCQ+AZ (0.6 on 1000 patients) and 11 among the 2114 who received other treatments $(p<0.0001)$. There were 9 deaths among the 1091 patients who received AZ alone $(0.82 \%)$ and 2 deaths among those who received no treatment. In the multivariable logistic regression, age, sex, and treatment, but not epidemic period, were associated with a significant difference in the risk of death (Table 2). $\mathrm{HCQ}+\mathrm{AZ}$ was associated with a significant $83 \%$ decrease in the risk of death $(0.17,0.06-0.48)$ independent of age, sex or epidemic period.

\subsubsection{Intensive care unit admissions}

Only 24 patients were transferred to the intensive care unit $(0.23 \%)$, with no patient under 40 years of age being transferred (Supplementary Table 4). In the multivariable logistic regression, age and sex were associated with ICU transfer (Supplementary Table 5). Period 3 was associated with a nonsignificant (aOR 0.44, 0.19-1.02) 66\% decrease in the risk of being transferred to the ICU independent of age, sex or $\mathrm{HCQ}+\mathrm{AZ}$ treatment. $\mathrm{HCQ}+\mathrm{AZ}$ was associated with a $44 \%$ nonsignificant $(0.56,0.24-1.30)$ decrease in the risk of ICU transfer (Supplementary Table 5). 


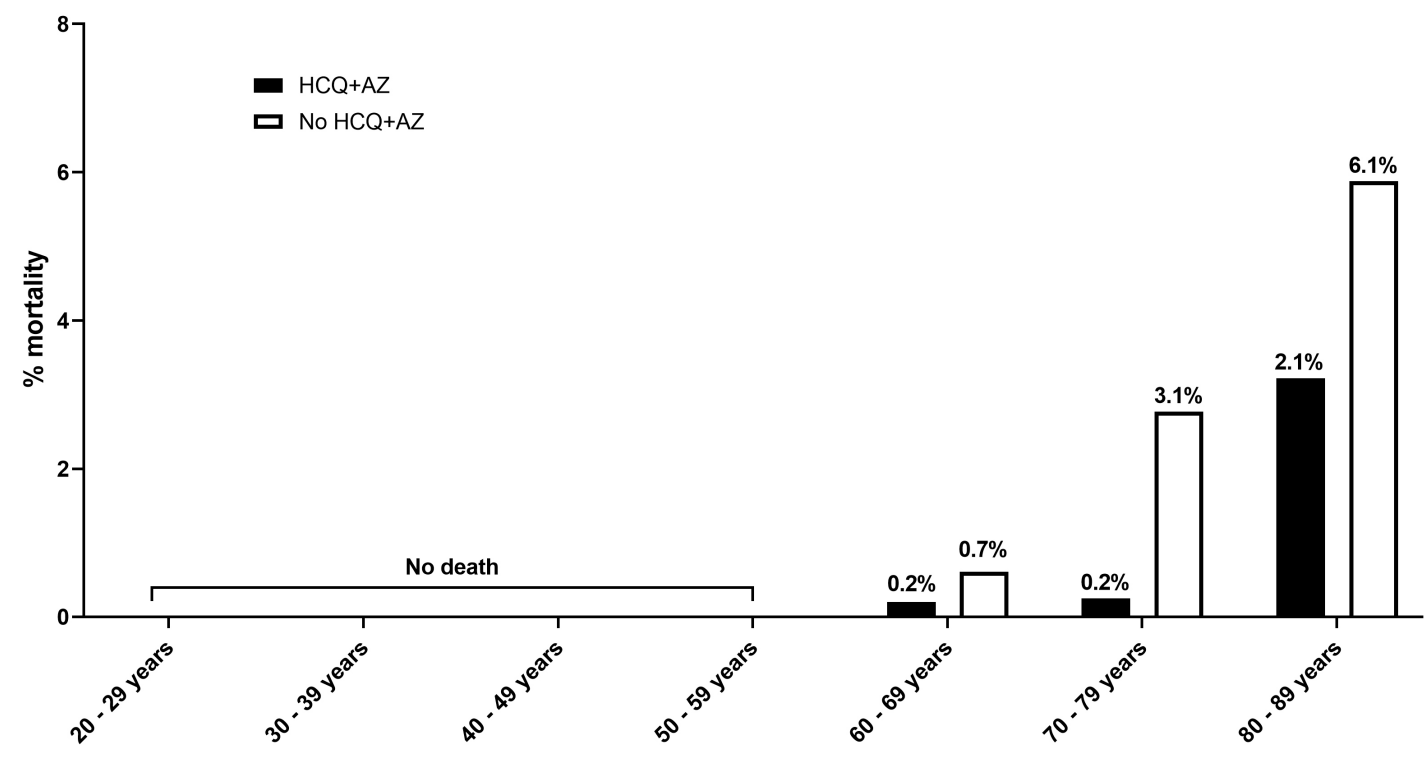

Fig. 3. Infection fatality rate by age class. HCQ+AZ, hydroxychloroquine and azithromycin treatment. There were only 16 patients $>89$ years, with no deaths in this cohort.

Table 1. Death rate according to treatment and age.

\begin{tabular}{|c|c|c|c|c|c|c|}
\hline & \multicolumn{2}{|c|}{ All } & \multicolumn{2}{|c|}{$\mathrm{HCQ}+\mathrm{AZ}$} & \multicolumn{2}{|c|}{ Other treatments } \\
\hline & $\mathrm{N}$ & $\%$ & $\mathrm{~N}$ & $\%$ & $\mathrm{n}$ & $\%$ \\
\hline $\mathrm{N}$ & $16 / 10,429^{\#}$ & 0.15 & $5 / 8315$ & 0.06 & $11 / 2114$ & 0.52 \\
\hline Male sex & $4832 / 10,429$ & 46.33 & $3914 / 8315$ & 47.07 & $918 / 2114$ & 43.42 \\
\hline \multicolumn{7}{|c|}{ Age interval (years) } \\
\hline $18-29$ & $0 / 2157$ & 0.00 & $0 / 1752$ & 0.00 & $0 / 405$ & 0.00 \\
\hline $30-39$ & $0 / 2004$ & 0.00 & $0 / 1650$ & 0.00 & $0 / 354$ & 0.00 \\
\hline $40-49$ & $0 / 2074$ & 0.00 & $0 / 1692$ & 0.00 & $0 / 382$ & 0.00 \\
\hline $50-59$ & $0 / 2179$ & 0.00 & $0 / 1726$ & 0.00 & $0 / 453$ & 0.00 \\
\hline$>59$ & $16 / 2015$ & 0.79 & $5 / 1495$ & 0.33 & $11 / 520$ & 2.21 \\
\hline $60-69$ & $4 / 1286$ & 0.31 & $2 / 1003$ & 0.20 & $2 / 283$ & 0.71 \\
\hline $70-79$ & $6 / 555$ & 1.08 & $1 / 395$ & 0.25 & $5 / 160$ & 3.13 \\
\hline $80-89$ & $6 / 158$ & 3.80 & $2 / 93$ & 2.15 & $4 / 65$ & 6.15 \\
\hline$>89$ & $0 / 16$ & 0.00 & $0 / 4$ & 0.00 & $0 / 12$ & 0.00 \\
\hline
\end{tabular}

HCQ, Hydroxychloroquine; AZ, Azithromycin.

\# An additional death occurred that was unrelated to COVID-19 or treatment but was not included in the analyses because no information can be described for forensic reasons.

\subsubsection{Hospitalizations}

Two hundred and seventy-eight patients $(2.7 \%)$ were subsequently hospitalized (Supplementary Table 6). In the multivariable logistic regression, age, sex, and epidemic period, but not $\mathrm{HCQ}+\mathrm{AZ}$, were associated with the hospitalization rate. The hospitalization rate was decreased by $30-35 \%$ for periods 2 and 3 compared with period 1 (Supplementary Table 7).

\section{Conclusions}

Here, we demonstrated the feasibility and efficacy of early outpatient management with a combination HCQ+AZ treatment to prevent COVID-19-related death. In our cohort, as in the largest published ambulatory series (Table 3, Ref.
[5, 6, 25-30]), treatment with HCQ was not associated with serious cardiac side effects but was associated with a significant IFR decrease of $75 \%$. The present cohort is among the largest cohorts of COVID-19 patients treated in the outpatient setting, with the lowest mortality rates: the IFR was $0.15 \%(0.06 \%$ among those treated with HCQ+AZ) versus $0.7 \%$ and $1.1 \%(0.30 \%$ and $0.39 \%$ among HCQ-treated patients) in the Iranian (169/22,784 patients with positive PCR) and Saudi ambulatory cohorts $(61 / 5541$ patients), respectively (Table 3) [5, 6].

In our cohort, the IFR among patients of all ages treated with HCQ+AZ was 60 per 100,000, which is much lower than the natural infection rate, even when evaluated under the best conditions, as in Iceland, where it was estimated to be 300 
Table 2. Effect of HCQ $+A Z$ on outpatient mortality-multivariable logistic regression $(n=2015$ patients $\geq 60$ years).

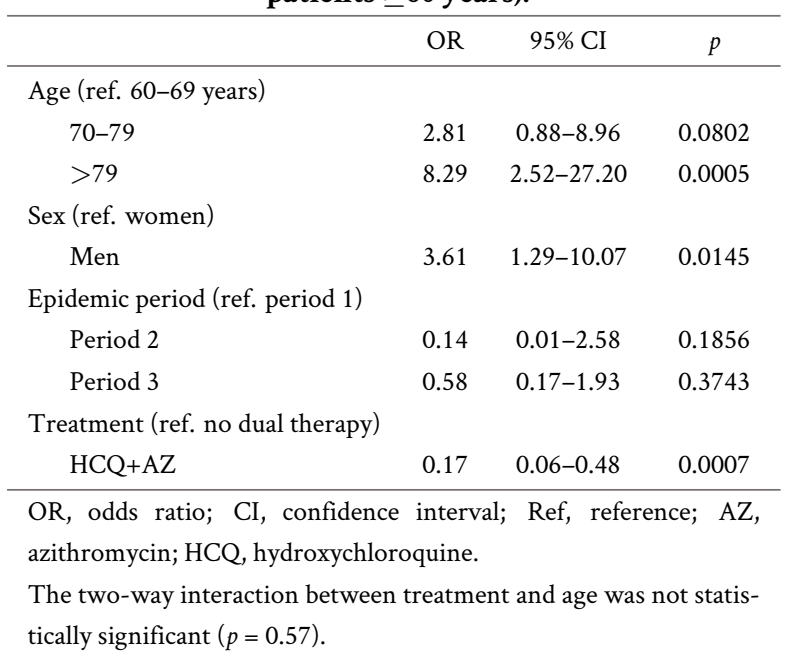

per 100,000 [31]. The IFR was also estimated to be 89 per 100,000 in patients who were $<70$ years of age in Denmark [32]. For the same age range in our cohort, the IFR was 41 per 100,000 (4/9700) for all ambulatory patients and 25 per 100,000 for those treated with HCQ+AZ (2/7823) (see Table 1).

The cardiotoxicity of HCQ, previously considered irrelevant to oral administration and usual doses [33], has been exaggerated by studies with a potential conflict of interest, notably in the retracted article published in the Lancet [34]. White [33] showed that the concentrations needed to inhibit the hERG channel responsible for QT prolongation were 4 to 14 times higher than the concentrations observed in plasma at usual doses. In our center, we developed a smartwatch electrocardiogram and artificial intelligence for assessing the cardiac rhythm safety of HCQ+AZ and did not find any QTc prolongation [35]. In the literature, ambulatory and not critically ill patients with COVID-19 treated with hydroxychloroquine, azithromycin and/or antiretrovirals develop a significant, but not relevant, QT interval prolongation [36]. As shown in our cohort, a simple clinical and biological evaluation with blood potassium assessment and the use of a first electrocardiogram allowed us to initiate treatment with acceptable safety in terms of potential arrhythmias.

The Fig. 4 show, in a meta-analysis, that all the studies carried out on outpatients, with minimal quality criteria (biological diagnosis, representative population with adequate control and at least 1 death), are all in the same direction (Table 3, Supplementary Tables 8,9, Supplementary Fig. 2). All these studies reported a similar magnitude (3-fold decrease in the risk of death), and showed that early treatment with hydroxychloroquine improve survival in COVID-19 outpatients in the community $(\mathrm{n}=32,124$ patients in 5 countries, Odds ratio $0.31[0.20-0.47])$ without heterogeneity $\left(\mathrm{I}^{2}=0 \%\right)$. This is also confirmed in the non-hospital setting in nursing homes (Table 3, Supplementary Table 8). Almost all
RCTs were excluded because of lack of systematic biological diagnosis or absence of death (Supplementary Table 9). Only 1 randomized controlled trial with PCR-proven diagnosis and at least 1 death was identified in the outpatient setting, probably because the sample size needed to identify a significant difference with sufficient power in mortality risk is difficult to achieve with such a design in this context. This RCT [27] reported a non-significant but beneficial effect of HCQ (Table 3). However, new RCTs with medical quality criteria are not expected to change results since it has been shown that results from RCT and observational studies did not differ significantly $[37,38]$. Interestingly, early administration of fluvoxamine was found to prevent clinical deterioration, notably in outpatients [29, 39]. Strikingly, both fluvoxamine and HCQ interfere with the interaction between the viral protein nsp6 and the host sigma-1 receptor, previously identified as a key functional host-dependency factor [40, 41].

The major limitation of the present cohort is its lack of assessment of comorbidities. Patients not receiving HCQ tend to be older and have more comorbidities [28], and although we were able to control for age and sex, the present study was not adjusted for other key comorbidities and may be biased accordingly. However, consistency with 3 similar studies assessing the death outcome and controlling for comorbidities $[5,6,25]$ was demonstrated by meta-analysis. In another study by Szente-Fonseca et al. [28], a beneficial effect of hydroxychloroquine, alone or in combination with prednisone, was found for hospitalization and death, but adjusted model was provided only for the hospitalization outcome. Taken together, these results suggest that early treatment with HCQ was consistently reported to prevent progression of COVID19 in ambulatory patients, independently of age, gender, and comorbidities.

All outpatients reported here were considered non-severe by the day-hospital physician based on routine assessment of saturation and dyspnea, but data on accurate initial clinical assessment was not collected. Follow-up was not systematically proposed after May 2020, so hospitalizations in and transfers to critical care units outside our city hospitals (APHM) may have been overlooked. However, deaths were identified through the French national register, thereby controlling this bias. The strengths of our study include the large sample size, the homogeneous management of patients associated with the monocentric design, and the double collection of death data by means of two registers: a local (city public hospital system) and national (French National Register of Deceased Persons) register.

The evolving concept and success of sequenced multidrug therapy including either HCQ, fluvoxamin or ivermectin for outpatient COVID-19 in reducing hospitalization and death by $\sim 85 \%$ was reported by several authors without any link of interest with our team which strengths the external validity of the present study (Table 3, Fig. 4) [2-4, 42]. In another non-hospital context, the efficacy of HCQ in nursing homes 
Table 3. Studies evaluating ambulatory treatments to prevent COVID-19 mortality ${ }^{a}$.

\begin{tabular}{|c|c|c|c|c|c|c|c|c|}
\hline \multirow{2}{*}{ Study } & \multirow{2}{*}{ Country } & \multirow{2}{*}{ Treatment } & \multirow{2}{*}{ Total sample size } & \multirow{2}{*}{ Treated } & \multirow{2}{*}{ Not treated } & \multirow{2}{*}{ Overall mortality/1000 } & Odds ratio $(95 \% \mathrm{CI})$ & \multirow{2}{*}{$p$-value } \\
\hline & & & & & & & $95 \% \mathrm{CI}$ & \\
\hline \multicolumn{9}{|l|}{ Studies controlling for age } \\
\hline Mokhtari et al., 2021 [5] & Iran & HCQ & $22,784^{b}$ & $5964^{b}$ & $16,820^{b}$ & 7.0 & aOR $0.33(0.21-0.55)$ & $0.0000082^{c}$ \\
\hline Present Study & France & $\mathrm{HCQ}+\mathrm{AZ}$ & 10,429 & $1495^{d}$ & $520^{d}$ & 1.5 & aOR $0.17(0.06-0.48)$ & 0.0007 \\
\hline Sulaiman et al., 2020 [6] & Saudi Arabia & HCQ & 5541 & 1817 & 3724 & 11.0 & aOR $0.36(0.16-0.80)$ & 0.012 \\
\hline Ip et al., 2021 [25] & USA & HCQ & 1274 & $97^{e}$ & $970^{e}$ & 40.0 & aOR $0.44(0.11-1.86)$ & 0.38 \\
\hline Lima-Morales et al., 2021 [26] & Mexico & IVM+AZ+Montelukast+Acetylsalicylic acid & 768 & 481 & 287 & 87.2 & aOR $0.19(0.10-0.36)$ & $<0.0001$ \\
\hline Reis et al., 2021 (RCT) [27] & Brazil & HCQ & 606 & 198 & 408 & 5.0 & Only 3 deaths in untreated & 0.30 \\
\hline \multicolumn{9}{|l|}{ Studies not controlling for age } \\
\hline Szente Fonseca et al., 2020 [28] & Brazil & HCQ & 717 & 334 & 383 & 15.3 & Unadj. OR $0.25(0.05-1.17)$ & $0.10^{f}$ \\
\hline Seftel et al., 2021 [29] & USA & Fluvoxamine & 113 & 65 & 48 & 8.8 & Only 1 death in untreated & $0.85^{f}$ \\
\hline
\end{tabular}

HCQ, hydroxychloroquine; AZ, azithromycin; IVM, ivermectin; RCT; randomized controlled trial; aOR, adjusted Odds ratio; 95\% CI, 95\% confidence interval.

${ }^{a}$ Only community studies are included. Studies in nursing homes are detailed in Supplementary Table 8. ${ }^{b}$ After exclusion of 5975 without positive PCR. ${ }^{c}$ Calculated from $95 \%$ confidence interval using the formula provided in Altman et al., 2011 [30]. ${ }^{d}$ Only 2015 patients $\geq 60$ years are included in the analysis because no death was observed in those younger than 60 years. ${ }^{e}$ Propensity-score-matched patients. ${ }^{f}$ Fisher exact test (calculated based on number provided in each article). Excluded studies (no death, absence of systematic PCR diagnosis) are listed in Supplementary Table 9. 


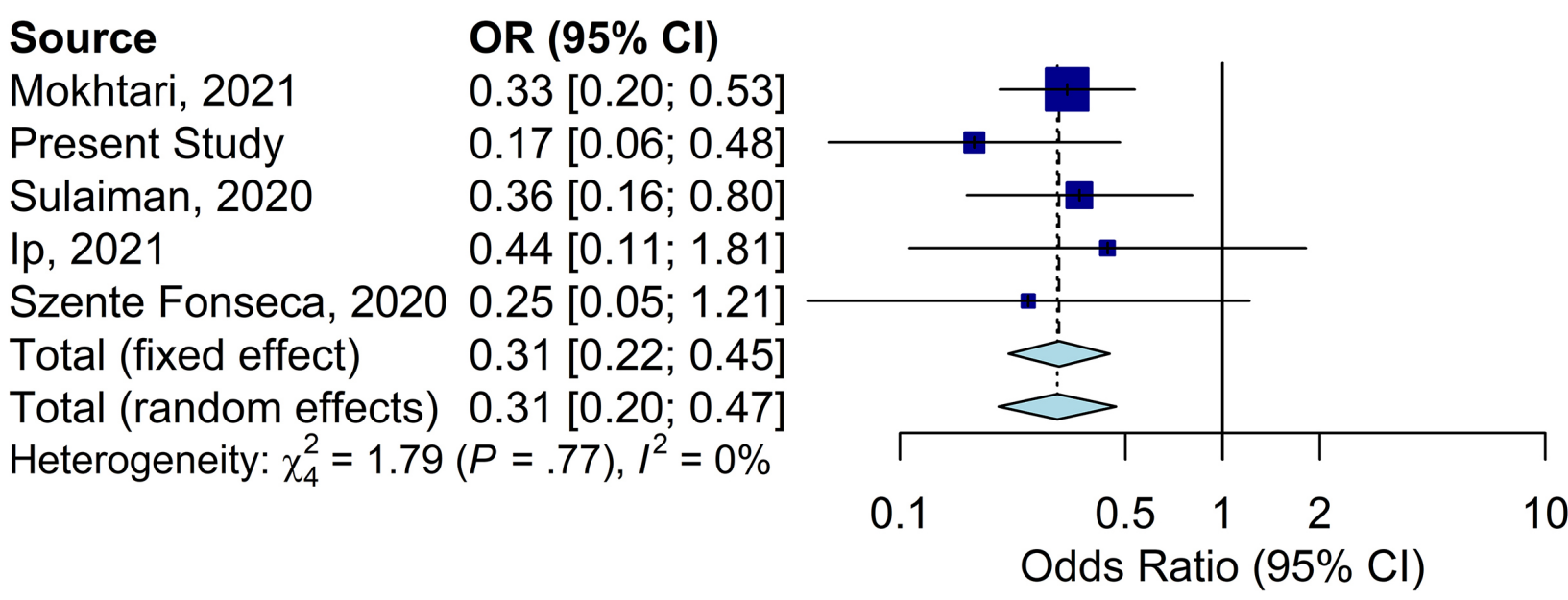

Fig. 4. Meta-analysis on studies in the community evaluating HCQ as an ambulatory treatment to prevent COVID-19 mortality (n $=32,124)$. The Meta-analysis was performed using random effects modeling for odds ratios (OR). Chi square based Q test and $\mathrm{I}^{2}$ statistic were used to evaluate the statistical heterogeneity between the studies. Meta-analysis was performed with using the R package meta.

(Supplementary Table 8) [43-45] strengthen the external validity and further highlight the role of HCQ in the nonhospital setting.

Finally, there are old and nontoxic drugs with in vitro and preliminary clinical efficacy on SARS-CoV-2 infection, such as HCQ, zinc, ivermectin, or fluvoxamine [2-4, 21, 39, 46]. Early oxygen, immunomodulation and antithrombotic therapy may also prevent disease worsening [3, 47]. Such drugs may be neglected when political factors, massive funding and fear lead to irrational decisions [48], such as therapeutic nihilism [2]. It seems urgent that governments and health authorities take in hand the evaluation of nonprofitable drugs, which are probably more effective than the drugs developed for this pandemic. This requires a profound paradigm shift, the extent of which was revealed by COVID-19 and which would be in line with the reflections on Tamiflu, recently documented in the British Medical Journal [48]. As long as the planned obsolescence of drugs continues and remains the current standard in Western countries, these richest countries and, theoretically, the most scientifically advanced, will remain those with the highest COVID-19 fatality rate in the world.

\section{Abbreviations \\ HCQ, hydroxychloroquine; AZ, azithromycin; IFR, infec- tion fatality rate.}

\section{Author contributions}

MMillion designed and supervised the study, conducted the investigation, formal analysis and wrote the first draft of the manuscript, JCL participated in the study design, investigation and critical review, all authors (HTD, IR, CD, CT, NC, SA, CA, KB, BD, SE, MH, MMailhe, CP, PS, CT, SG, EJ, AGG, HC, LCJ, PC, PG, PEF, BM, JCD, PH, JYG, AJ, $\mathrm{SH}, \mathrm{KGL}, \mathrm{YO}, \mathrm{PP}, \mathrm{PB}$ ) participated in the investigation and provided constructive criticism and comments, LD, CB and SC conducted the formal analysis and meta-analysis, DR designed and supervised the study, and final proofreading with key finalization of the text.

\section{Ethics approval and consent to participate}

Data presented herein were collected retrospectively from the routine care setting using the electronic health recording system of the hospital. The retrospective nature of the study was approved by our institutional review board committee (Méditerranée Infection $\mathrm{N}^{\circ}$ : 2021-007). As previously reported [15], for all patients, the prescription of HCQ+AZ was made during day hospital attendance by one of the physicians, after a collegial decision based on the most recent scientific data available and after assessment of the benefit/harm ratio of the treatment. According to European General Data Protection Regulation No 2016/679, patients were informed of the potential use of their medical data and that they could refuse the use of their data. Data processing was carried out in accordance with the reference methodology MR-004 registered on $\mathrm{N}^{\circ}$ MR 2020-151 in the AP-HM register and public register (https://www.health-data-hub.fr/projets).

\section{Acknowledgment}

This manuscript has been edited by a native English speaker. We thank the reviewers who helped in substantially improving and clarifying the manuscript with their many comments and suggestions. We are thankful to Sylvie Arlotto, Marion Bechet, Yacine Belkhir, Pierre Dudouet, Véronique Filosa, Marie-Thérèse Jimeno, Alexandra Kotovtchikhine, Line Meddeb, Cléa Melenotte, Malika Mokhtari, and Pierre Pinzelli. All medical students from Aix Marseille University; all nurses; all laboratory staff; all administrative, technical and security staff from Assistance Publique-Hôpitaux de Marseille and IHU Méditerranée In- 
fection; all volunteer medical doctors; and the Bataillon des Marins Pompiers de Marseille for their help. Other medical volunteers who contributed to taking care of COVID-19 patients in 2020 are acknowledged at the end of the Supplementary data.

\section{Funding}

This work was funded by ANR-15-CE36-0004-01 and by ANR "Investissements d'avenir", Méditerranée infection 10IAHU-03 and was also supported by Région Provence-AlpesCôte d'Azur. This work received financial support from the Mediterranean Infection Foundation.

\section{Conflict of interest}

The authors declare no conflict of interest. The funders/sponsors had no role in the design and conduct of the study; collection, management, analysis, and interpretation of the data; preparation, review, or approval of the manuscript; or decision to submit the manuscript for publication. Funding sources had no role in the design and conduct of the study; collection, management, analysis, and interpretation of the data; and preparation, review, or approval of the manuscript. Our group used widely available generic drugs distributed by many pharmaceutical companies.

\section{Supplementary material}

Supplementary material associated with this article can be found, in the online version, at https://rcm.imrpress.com/E N/10.31083/j.rcm2203116.

\section{References}

[1] Worldometer. COVID-19 Coronavirus pandemic. 2021. Available at: https://www.worldometers.info/coronavirus/ (Accessed: 4 August 2021).

[2] McCullough PA, Alexander PE, Armstrong R, Arvinte C, Bain $\mathrm{AF}, \mathrm{Bartlett} \mathrm{RP}$, et al. Multifaceted highly targeted sequential multidrug treatment of early ambulatory high-risk SARS-CoV-2 infection (COVID-19). Reviews in Cardiovascular Medicine. 2020; 21: 517-530.

[3] McCullough PA, Kelly RJ, Ruocco G, Lerma E, Tumlin J, Wheelan $\mathrm{KR}$, et al. Pathophysiological Basis and Rationale for Early Outpatient Treatment of SARS-CoV-2 (COVID-19) Infection. The American Journal of Medicine. 2021; 134: 16-22.

[4] Procter BC, Ross C, Pickard V, Smith E, Hanson C, McCullough PA. Clinical outcomes after early ambulatory multidrug therapy for high-risk SARS-CoV-2 (COVID-19) infection. Reviews in Cardiovascular Medicine. 2020; 21: 611-614.

[5] Mokhtari M, Mohraz M, Gouya MM, Namdari Tabar H, Tabrizi $\mathrm{J}$, Tayeri K, et al. Clinical outcomes of patients with mild COVID19 following treatment with hydroxychloroquine in an outpatient setting. International Immunopharmacology. 2021; 96: 107636.

[6] Sulaiman T, Mohana A, Alawdah L, Mahmoud N, Hassanein M, Wani $\mathrm{T}$, et al. The Effect of Early Hydroxychloroquine-based Therapy in COVID-19 Patients in Ambulatory Care Settings: A Nationwide Prospective Cohort Study. medRxiv. 2020.

[7] Su Y, Ling Y, Ma Y, Tao L, Miao Q, Shi Q, et al. Efficacy of early hydroxychloroquine treatment in preventing COVID-19 pneumonia aggravation, the experience from Shanghai, China. Bioscience Trends. 2021; 14: 408-414.

[8] Gangopadhyay KK, Sinha B, Ghosal S. "Compliance of the Indian National Task Force guidelines for COVID-19 recommendation by Indian doctors - a survey". Diabetes \& Metabolic Syndrome. 2020; 14: 1413-1418.

[9] Izoulet M. National consumption of antimalarial drugs and COVID-19 deaths dynamics: An econometric study. Journal of Clinical Toxicology. 2020; 10: 456.

[10] Liu J, Cao R, Xu M, Wang X, Zhang H, Hu H, et al. Hydroxychloroquine, a less toxic derivative of chloroquine, is effective in inhibiting SARS-CoV-2 infection in vitro. Cell Discovery. 2020; 6: 16.

[11] Wang M, Cao R, Zhang L, Yang X, Liu J, Xu M, et al. Remdesivir and chloroquine effectively inhibit the recently emerged novel coronavirus (2019-nCoV) in vitro. Cell Research. 2020; 30: 269271.

[12] Gautret P, Lagier J, Parola P, Hoang VT, Meddeb L, Mailhe M, et al. Hydroxychloroquine and azithromycin as a treatment of COVID-19: results of an open-label non-randomized clinical trial. International Journal of Antimicrobial Agents. 2020; 56: 105949.

[13] Andreani J, Le Bideau M, Duflot I, Jardot P, Rolland C, Boxberger $\mathrm{M}$, et al. In vitro testing of combined hydroxychloroquine and azithromycin on SARS-CoV-2 shows synergistic effect. Microbial Pathogenesis. 2020; 145: 104228.

[14] Gautret P, Million M, Jarrot P, Camoin-Jau L, Colson P, Fenollar F, et al. Natural history of COVID-19 and therapeutic options. Expert Review of Clinical Immunology. 2020; 16: 1159-1184.

[15] Lagier J, Million M, Gautret P, Colson P, Cortaredona S, GiraudGatineau A, et al. Outcomes of 3,737 COVID-19 patients treated with hydroxychloroquine/azithromycin and other regimens in Marseille, France: a retrospective analysis. Travel Medicine and Infectious Disease. 2020; 36: 101791.

[16] Jaafar R, Aherfi S, Wurtz N, Grimaldier C, Van Hoang T, Colson $\mathrm{P}$, et al. Correlation between 3790 Quantitative Polymerase Chain Reaction-Positives Samples and Positive Cell Cultures, Including 1941 Severe Acute Respiratory Syndrome Coronavirus 2 Isolates. Clinical Infectious Diseases. 2021; 72: e921.

[17] Amrane S, Tissot-Dupont H, Doudier B, Eldin C, Hocquart M, Mailhe $M$, et al. Rapid viral diagnosis and ambulatory management of suspected COVID-19 cases presenting at the infectious diseases referral hospital in Marseille, France, - January 31st to March 1st, 2020: a respiratory virus snapshot. Travel Medicine and Infectious Disease. 2020; 36: 101632.

[18] Fournier P, Zandotti C, Ninove L, Prudent E, Colson P, Gazin $\mathrm{C}$, et al. Contribution of VitaPCR SARS-CoV-2 to the emergency diagnosis of COVID-19. Journal of Clinical Virology. 2020; 133: 104682.

[19] Tang N, Bai H, Chen X, Gong J, Li D, Sun Z. Anticoagulant treatment is associated with decreased mortality in severe coronavirus disease 2019 patients with coagulopathy. Journal of Thrombosis and Haemostasis. 2020; 18: 1094-1099.

[20] Ayerbe L, Risco C, Ayis S. The association between treatment with heparin and survival in patients with Covid-19. Journal of Thrombosis and Thrombolysis. 2020; 50: 298-301.

[21] Carlucci PM, Ahuja T, Petrilli C, Rajagopalan H, Jones S, Rahimian J. Zinc sulfate in combination with a zinc ionophore may improve outcomes in hospitalized COVID-19 patients. Journal of Medical Microbiology. 2020; 69: 1228-1234.

[22] Colson P, Levasseur A, Gautret P, Fenollar F, Thuan Hoang V, Delerce J, et al. Introduction into the Marseille geographical area of a mild SARS-CoV-2 variant originating from sub-Saharan Africa: an investigational study. Travel Medicine and Infectious Disease. 2021; 40: 101980.

[23] Fournier P, Colson P, Levasseur A, Devaux CA, Gautret P, Bedotto M, et al. Emergence and outcomes of the SARS-CoV-2 'Marseille-4' variant. International Journal of Infectious Diseases. 2021; 106: 228-236.

[24] Institut National de la Statistique et des Etudes Economiques (INSEE). French national register of deceased persons. Year. Available at: https://www.data.gouv.fr/fr/datasets/fichier-des-perso nnes-decedees/\#_(Accessed: 1 March 2021). 
[25] Ip A, Ahn J, Zhou Y, Goy AH, Hansen E, Pecora AL, et al. Hydroxychloroquine in the treatment of outpatients with mildly symptomatic COVID-19: a multi-center observational study. BMC Infectious Diseases. 2021; 21: 72.

[26] Lima-Morales R, Méndez-Hernández P, Flores YN, OsornoRomero P, Sancho-Hernández CR, Cuecuecha-Rugerio E, et al. Effectiveness of a multidrug therapy consisting of Ivermectin, Azithromycin, Montelukast, and Acetylsalicylic acid to prevent hospitalization and death among ambulatory COVID-19 cases in Tlaxcala, Mexico. International Journal of Infectious Diseases. 2021; 105: 598-605.

[27] Reis G, Moreira Silva EADS, Medeiros Silva DC, Thabane L, Singh G, Park JJH, et al. Effect of Early Treatment with Hydroxychloroquine or Lopinavir and Ritonavir on Risk of Hospitalization among Patients with COVID-19. JAMA Network Open. 2021; 4: e216468.

[28] Szente Fonseca SN, de Queiroz Sousa A, Wolkoff AG, Moreira MS, Pinto BC, Valente Takeda CF, et al. Risk of hospitalization for Covid-19 outpatients treated with various drug regimens in Brazil: Comparative analysis. Travel Medicine and Infectious Disease. 2020; 38: 101906.

[29] Seftel D, Boulware DR. Prospective Cohort of Fluvoxamine for Early Treatment of Coronavirus Disease 19. Open Forum Infectious Diseases. 2021; 8: ofab050.

[30] Altman DG, Bland JM. How to obtain the P value from a confidence interval. British Medical Journal. 2011; 343: d2304.

[31] Gudbjartsson DF, Norddahl GL, Melsted P, Gunnarsdottir K, Holm H, Eythorsson E, et al. Humoral Immune Response to SARSCoV-2 in Iceland. New England Journal of Medicine. 2020; 383: 1724-1734.

[32] Erikstrup C, Hother CE, Pedersen OBV, Mølbak K, Skov RL, Holm DK, et al. Estimation of SARS-CoV-2 Infection Fatality Rate by Real-time Antibody Screening of Blood Donors. Clinical Infectious Diseases. 2021; 72: 249-253.

[33] White NJ. Cardiotoxicity of antimalarial drugs. The Lancet Infectious Diseases. 2007; 7: 549-558.

[34] Mehra MR, Desai SS, Ruschitzka F, Patel AN. RETRACTED: Hydroxychloroquine or chloroquine with or without a macrolide for treatment of COVID-19: a multinational registry analysis. The Lancet. 2020; S0140-6736(20)31180-6.

[35] Maille B, Wilkin M, Million M, Rességuier N, Franceschi F, Koutbi-Franceschi L, et al. Smartwatch Electrocardiogram and Artificial Intelligence for Assessing Cardiac-Rhythm Safety of Drug Therapy in the COVID-19 Pandemic. The QT-logs study. International Journal of Cardiology. 2021; 331: 333-339.

[36] Jiménez-Jáimez J, Macías-Ruiz R, Bermúdez-Jiménez F, RubiniCosta R, Ramírez-Taboada J, Flores PIG, et al. Absence of relevant QT interval prolongation in not critically ill COVID-19 patients. Scientific Reports. 2020; 10: 21417.
[37] Anglemyer A, Horvath HT, Bero L. Healthcare outcomes assessed with observational study designs compared with those assessed in randomized trials. The Cochrane Database of Systematic Reviews. 2014; 2014: MR000034.

[38] Concato J, Shah N, Horwitz RI. Randomized, Controlled Trials, Observational Studies, and the Hierarchy of Research Designs. New England Journal of Medicine. 2000; 342: 1887-1892.

[39] Lenze EJ, Mattar C, Zorumski CF, Stevens A, Schweiger J, Nicol GE, et al. Fluvoxamine vs Placebo and Clinical Deterioration in Outpatients with Symptomatic COVID-19: A Randomized Clinical Trial. Journal of the American Medical Association. 2020; 324: 2292-2300.

[40] Gordon DE, Jang GM, Bouhaddou M, Xu J, Obernier K, White $\mathrm{KM}$, et al. A SARS-CoV-2 protein interaction map reveals targets for drug repurposing. Nature. 2020; 583: 459-468.

[41] Gordon DE, Hiatt J, Bouhaddou M, Rezelj VV, Ulferts S, Braberg $\mathrm{H}$, et al. Comparative host-coronavirus protein interaction networks reveal pan-viral disease mechanisms. Science. 2020; 370: eabe9403.

[42] Ladapo JA, McKinnon JE, McCullogh PA, Risch HA. Randomized Controlled Trials of Early Ambulatory Hydroxychloroquine in the Prevention of COVID-19 Infection, Hospitalization, and Death: Meta-Analysis. medRxiv. 2020.

[43] Cangiano B, Fatti LM, Danesi L, Gazzano G, Croci M, Vitale G, et al. Mortality in an Italian nursing home during COVID-19 pandemic: correlation with gender, age, $\mathrm{ADL}$, vitamin $\mathrm{D}$ supplementation, and limitations of the diagnostic tests. Aging. 2020; 12: 24522-24534.

[44] Heras E, Garibaldi P, Boix M, Valero O, Castillo J, Curbelo Y, et al. COVID-19 mortality risk factors in older people in a long-term care center. European Geriatric Medicine. 2021; 12: 601-607.

[45] Ly TDA, Zanini D, Laforge V, Arlotto S, Gentile S, Mendizabal H, et al. Pattern of SARS-CoV-2 infection among dependant elderly residents living in long-term care facilities in Marseille, France, March-June 2020. International Journal of Antimicrobial Agents. 2020; 56: 106219.

[46] Rajter JC, Sherman MS, Fatteh N, Vogel F, Sacks J, Rajter J. Use of Ivermectin is Associated with Lower Mortality in Hospitalized Patients with Coronavirus Disease 2019. Chest. 2021; 159: 85-92.

[47] Long L, Wu L, Chen L, Zhou D, Wu H, Lu D, et al. Effect of early oxygen therapy and antiviral treatment on disease progression in patients with COVID-19: A retrospective study of medical charts in China. PLoS Neglected Tropical Diseases. 2021; 15: e0009051.

[48] Godlee F. Covid-19: The lost lessons of Tamiflu. British Medical Journal. 2020; 371: m4701. 